\title{
Numerical Simulations of Chromospheric Microflares
}

\author{
R. L. Jiang, C. Fang and P. F. Chen \\ Department of Astronomy, Nanjing University, Nanjing 210093, China \\ Email: fangc@nju.edu.cn
}

Received __; accepted _ 


\begin{abstract}
With gravity, ionization, and radiation being considered, we perform 2.5D compressible resistive MHD simulations of chromospheric magnetic reconnection using the CIP-MOCCT scheme. The temperature distribution of the quiet-Sun atmospheric model VALC and the helium abundance (10\%) are adopted. Our 2.5D MHD simulation reproduces qualitatively the temperature enhancement observed in chromospheric microflares. The temperature enhancement $\Delta T$ is demonstrated to be sensitive to the background magnetic field, whereas the total evolution time $\Delta t$ is sensitive to the magnitude of the anomalous resistivity. Moveover, we found a scaling law, which is described as $\Delta T / \Delta t \sim$ $n_{H}{ }^{-1.5} B^{2.1} \eta_{0}{ }^{0.88}$. Our results also indicate that the velocity of the upward jet is much greater than that of the downward jet and the X-point may move up or down.
\end{abstract}

Subject headings: MHD —Sun: activity_-Sun: chromosphere - Sun: flares

\title{
1. INTRODUCTION
}

Magnetic reconnection plays a very important role in solar flares, corona mass ejections, and other solar activities. During the solar minimum, many authors focus their attention on the solar small-scale activities such as microflares (Qiu et al. 2004; Fang et al. 2006a; Ning 2008; Brosius \& Holman 2009), Ellerman bombs (Fang et al. 2006b; Watanabe et al. 2008), chromospheric jets (Nishizuka et al. 2008), and so on.

Microflares, or subflares, or bright points, are small-scale and short-lifetime solar activities. The size of microflares ranges from several arcsecs to about 20 arcsecs, the duration and the total energy can be $10-30$ minutes and $10^{26}-10^{29}$ ergs, respectively 
(Shimizu et al. 2002; Fang et al. 2006a). The most distinctive feature in their visible spectra is the faint emission at the center and the brightening at the wing of some chromospheric 1984), EUV (Porter et al. 1984; Emslie \& Noyes 1978) and microwave (Gary \& Zirin 1988) emissions have also been observed in some microflares. It is an interesting fact that there are some correlations among the emissions at different wavelengths. For instance, Qiu et al. (2004) found that about $40 \%$ of microflares show HXR emissions at $>10 \mathrm{keV}$ and microwave emissions at $\sim 10 \mathrm{GHz}$. Recently, Ning (2008) found that there is a correlation between the power-law index of the HXR spectrum and the emission measure of microflares. Brosius \& Holman (2009) found that the studied microflare is bright first in the chromospheric and transition region spectral lines rather than in the corona, which is consistent with the chromospheric heating by nonthermal electron beams. All these evidence indicates that microflares are related to the nonthermal processes driven by magnetic reconnection.

Some microflares might result from the magnetic reconnection in the corona. However, there is accumulating evidence suggesting that some other microflares are due to the reconnection in the chromosphere (Liu et al. 2004; Xia et al. 2007). For instance, Tang et al. (2000) found that in many cases emerging flux occurred about 5 - 30 minutes before the microflares, where the emerging flux may collide with the pre-existing magnetic field at the chromospheric height. Brosius \& Holman (2009) also stated that magnetic reconnection in the chromosphere could be a plausible mechanism for triggering the chromospheric microflare.

Chen et al. (2001) made 2.5-dimensional (2.5D) numerical simulations of chromospheric magnetic reconnection in order to study Ellerman bombs and type II white-light flares. However, in the simulations, they made some simplifications, such as the omissionof 
the gravity. In this paper, in order to improve their results, we perform 2.5D magnetohydrodynamic (MHD) simulations using the CIP-MOCCT scheme, with gravity being included, and try to make some comparisons with the observations of chromospheric microflares. In the next section, the numerical method is given in detail. Our results, which include the dynamic process, parameter dependence, a scaling law and a comparison with a semi-empirical model, are described in Section 3. Discussion and summary are given in Section 4

\section{NUMERICAL METHOD}

\subsection{Basic Equations}

In our simulation, we solve $2.5 \mathrm{D}$ compressible resistive $\mathrm{MHD}$ equations with ionization, radiation, and uniform gravitational field being considered. There is no thermal conduction in our simulation since thermal conductivity is very small in the chromosphere. The MHD equations are given as follows:

$$
\begin{gathered}
\frac{\partial \rho}{\partial t}+\nabla \cdot(\rho \mathbf{v})=0 \\
\frac{\partial(\rho \mathbf{v})}{\partial t}+\nabla \cdot\left[\left(p+\frac{B^{2}}{2 \mu_{0}}\right) \mathbf{I}+\rho \mathbf{v} \mathbf{v}-\frac{1}{\mu_{0}} \mathbf{B B}\right]=\rho \mathbf{g}, \\
\frac{\partial \mathbf{B}}{\partial t}+\nabla \cdot(\mathbf{v B}-\mathbf{B v})=-\nabla \times\left(\frac{\eta}{\mu_{0}} \nabla \times \mathbf{B}\right), \\
\frac{\partial e}{\partial t}+\nabla \cdot\left[\mathbf{v}\left(e+\frac{B^{2}}{2 \mu_{0}}+p\right)-\frac{1}{\mu_{0}} \mathbf{B}(\mathbf{B} \cdot \mathbf{v})+\left(\frac{\eta}{\mu_{0}^{2}} \nabla \times \mathbf{B}\right) \times \mathbf{B}\right]=\rho \mathbf{g} \cdot \mathbf{v}+H-R_{r},
\end{gathered}
$$


where eight independent variables are the density $(\rho)$, velocity $\left(v_{x}, v_{y}, v_{z}\right)$, magnetic field $\left(B_{x}, B_{y}, B_{z}\right)$, and temperature $(T)$. The thermal and ionization terms are included into the total energy density $e=p /(r-1)+\rho v^{2} / 2+B^{2} / 2 \mu_{0}+\chi_{H} n_{e}$, where $\chi_{H}$ is the ionization potential of hydrogen atoms and $\mu_{0}$ is the vacuum permeability. Considering the helium abundance $(10 \%)$, we take $\rho=1.4 m_{H} n_{H}$, where $n_{H}$ is the number density of hydrogen. With partial ionization considered, the total gas pressure is expressed as $p=\left(1.1 n_{H}+n_{e}\right) k_{B} T$, where $k_{B}$ is the Boltzmann constant and the number density of electrons, $n_{e}$, is deduced from the modified Saha and Boltzmann formula for a pure hydrogen atmosphere (Gan \& Fang 1990):

$$
\begin{gathered}
n_{e}=\left(\sqrt{\phi^{2}+4 n_{H} \phi}-\phi\right) / 2, \\
\phi=\frac{1}{b_{1}}\left(\frac{2 \pi m_{e} \kappa_{B} T}{h_{0}^{2}}\right)^{3 / 2} e^{\frac{-\chi_{H}}{\kappa_{B} T}}, \\
b_{1}=\frac{2 T}{T_{R}} e^{\frac{\chi_{H}}{4 \kappa_{B} T}\left(\frac{T}{T_{R}}-1\right)},
\end{gathered}
$$

where $h_{0}$ is the Planck constant. We take $T_{R}=6000 \mathrm{~K}$ (Brown 1973). In Equation (4), $R_{r}$ and $H$ are the radiative loss and the pre-heating term, respectively. Radiative loss is important in the chromosphere and the photosphere. Generally speaking, it should be calculated by solving simultaneously the statistical equilibrium and radiative transfer equations along with the MHD equations. However, it is not feasible to deal with it in our $2.5 \mathrm{D}$ numerical simulation. Instead, we fit the non-local thermodynamic equilibrium (non-LTE) radiative losses in the VALC model for the quiet-Sun atmosphere (Vernazza et al. 1981) and the F1 semi-empirical model for the weak flare (Machado et al. 1980), and then modify the empirical formula given by Gan \& Fang (1990) in order to make 
it more suitable for the small scale activities such as microflares:

$$
\begin{gathered}
R_{r}=n_{H} n_{e} \alpha(y) f(T), \\
\alpha(y)=10^{\left(1.745 \times 10^{-3} y-4.739\right)}+8.0 \times 10^{-2} e^{-3.701 \times 10^{-2} y}, \\
f(T)=4.533 \times 10^{-23}\left(\frac{T}{10^{4}}\right)^{2.874},
\end{gathered}
$$

where $\alpha(y)$ and $f(T)$ are functions of $y$ (the height from $\tau_{5000}=1$ in the photosphere) and the temperature $T$, respectively. The unit of $y$ is $\mathrm{km}$. The suitable range of the height and the temperature in Equation (8) is $h<2000 \mathrm{~km}$ and $T<10^{5} \mathrm{~K}$, respectively. The pre-heating term is given by $H=n_{H}\left(n_{e} \alpha f\right)_{t=0}$, which balances the radiative loss before magnetic reconnection.

\subsection{Normalization and Parameters}

In order to non-dimensionalize the MHD Equations (11) - (41) in Section 2.1, the variables are normalized by the quantities given in Table 1. In this table, $m_{H}$ is the proton mass. The dimensionless MHD Equations, which have a standard form of advection and non-advection terms, are numerically solved with the CIP-MOCCT scheme (Kudoh 1999). Besides the acoustic transit time scale $t_{0}$ used in the nondimensionalization, another practical time scale is the Alfvén transit time $\tau_{A}=L_{0} / v_{A}$, where $v_{A}$ is the Alfvén velocity at the height of the reconnection X-point. In our simulations, two cases are considered, where the magnetic reconnection X-point is set at two different heights, i.e., $500 \mathrm{~km}$ and $1000 \mathrm{~km}$. The corresponding Alfvén velocities are $18.2 \mathrm{~km} \mathrm{~s}^{-1}$ and $11.2 \mathrm{~km} \mathrm{~s}^{-1}$, while the 
corresponding Alfvén transit time is 109.9 s and 177.6 s, respectively.

\subsection{Initial and Boundary Conditions}

The domain of the numerical simulation is $-1000 \mathrm{~km} \leq x \leq 1000 \mathrm{~km}, 0 \leq y \leq 2000$ $\mathrm{km}$, as shown in Figure 1. The initial magnetic configuration is a force-free field with a current sheet located along the $y$-axis, which was used by Chen et al. (1999) and rewritten as follows:

$$
\begin{gathered}
B_{x}=0, \\
B_{y}= \begin{cases}B_{0} \frac{x}{|x|} & |x|>\Delta h \\
B_{0} \sin \frac{x \pi}{2 \triangle h} & |x|<\Delta h,\end{cases} \\
B_{z}= \begin{cases}0 & |x|>\Delta h \\
B_{0} \cos \frac{x \pi}{2 \Delta h} & |x|<\Delta h,\end{cases}
\end{gathered}
$$

where $B_{0}$ and $\triangle h$ are the background magnetic field and the half width of current sheet, respectively. In order to make our simulation more realistic, we use the temperature distribution of the quiet-Sun atmospheric model VALC as shown in Figure 2 (left panel). Using the hydrostatic equilibrium equation and the modified Saha and Boltzmann formula for calculating the number density of electrons iteratively, we get the initial number density of hydrogen $\left(n_{H}\right)$ and electrons $\left(n_{e}\right)$ as shown in the middle and the right panels of Figure 2. respectively. From these figures we can see that our initial model is very close to the VALC model. In order to initiate the magnetic reconnection, we assume that an anomalous resistivity (Cramer \& Donnelly 1979), with the form $\eta=\eta_{0} \cos [x \pi / 2 \triangle h] \cos \left[\left(y-h_{r}\right) \pi / 2 \triangle h\right]$, is localized in a small region $|x| \leq \triangle h,\left|y-h_{r}\right| \leq \triangle h$ (the small rectangle in Figures 1, 7 
and 12), where $h_{r}$ stands for the height of the reconnection point (X-point) in the $y$-axis, $\triangle h$ is the half width of the resistivity region and $\eta_{0}$ is the resistive amplitude. It is noted that the half width of the resistivity region is taken to be the same as the half width of the current sheet.

Owing to the symmetry, the calculation is performed only in the right half region, with the symmetry boundary condition being set on the left-hand side. The right and the upper boundaries are treated as open boundaries. Line-tying conditions are applied to the bottom boundary. It is noted that $\rho$ should satisfy the hydrostatic equilibrium $(\partial p / \partial y+\rho g=0)$ to reach a balance between the gravity and the pressure gradient force at the upper and the lower boundaries. The calculation domain is discretized into $800 \times 400$ grid meshes. The grid sizes are $\triangle x=1.25 \mathrm{~km}$ and $\triangle y=5 \mathrm{~km}$, which are much less than the pressure scale height $(>100 \mathrm{~km})$. With gravity, ionization and radiation being considered, and with heat conduction being neglected, the numerical simulations are performed with the CIP-MOCCT scheme.

\section{NUMERICAL RESULTS}

\subsection{Dynamic Process}

Generally speaking, the height of the X-point may change as the reconnection proceeds

(Takeuchi \& Shibata 2001; von Rekowski \& Hood 2008). In our simulation, we found that the X-point could move up or down by $10-40 \mathrm{~km}$, but still within the resistivity region. For this reason, we do not need to change the position of the resistivity region. In order to illustrate the dynamic process, this subsection describes the simulation results in a typical case, where the background magnetic field $B_{0}$ is taken to be $50 \mathrm{G}$, the anomalous resistivity $\eta_{0}$ be $17.7 \Omega \mathrm{m}$, the X-point height $h_{r}$ be $1000 \mathrm{~km}$, and the half width of the resistivity 
region $\triangle h$ be $100 \mathrm{~km}$. Figure 3 depicts the results of the simulation at different times. In this figure the color stands for the temperature, solid lines for magnetic field and arrows for velocity.

As the anomalous resistivity sets in, we have a fast reconnection process similar to that predicted by Petschek (1964). Figure 4 shows the evolution of the reconnection rate, which is defined as $\left|v_{i n}\right| / v_{A}$, where $v_{\text {in }}$ is the maximum inflow velocity along the $x$-direction at the height of the X-point. It is found that the simulation stops at $t=1.35 \tau_{A}(148.2 \mathrm{~s})$, probably due to some numerical instability. The reconnection rate reaches the maximum, 0.11 , at $t=1.1 \tau_{A}(120.8 \mathrm{~s})$. After that, the reconnection rate remains almost constant around 0.1. The lifetime of the reconnection simulated in this case (148.2 s) is shorter than the typical lifetime of microflares, i.e., 10 - 30 minutes. Maybe our results can only represent the pre-flare phase and the rise phase.

During the reconnection, the temperature enhancement in the resistivity region is about $2000-3000 \mathrm{~K}$ as shown in Figure 5. Accelerated by the magnetic tension force, the hot plasma together with the frozen-in reconnected field lines are ejected from the resistivity region to form upward and downward reconnection jets. The maximum velocity of the upward and downward jets are about $V_{u p}=23.0 \mathrm{~km} \mathrm{~s}^{-1}$ and $V_{\text {down }}=9.4 \mathrm{~km} \mathrm{~s}^{-1}$, respectively as shown in Figure 6. Because of the sharp density gradient in the lower atmosphere, the speed of the upward jet is faster than that of the downward one. Compared with the local Alfvén speed $\left(V_{A}=18.2 \mathrm{~km} \mathrm{~s}^{-1}\right)$ at the height of $1000 \mathrm{~km}$, we find that the velocity of the outflow is approximately the local Alfvén speed in the inflow region. The downward jet can only reach the height of $600 \mathrm{~km}$, after which it is slowed down significantly. Furthermore, the reconnection X-point also rises by about $40 \mathrm{~km}$ during the reconnection process.

The hot plasma jet ejected along the $y$-axis is mainly heated by the Joule dissipation 
in the resistivity region. However, we can not ignore the heating by the slow-mode shocks. In our simulation, the heating occurs not only in the resistivity region but also outside the region as shown in Figure 7, which shows the current density (z-component) distribution in the left panel and the temperature distribution in the right panel. It can be seen that a pair of slow-mode shocks, which are characterized by the high current density, are formed at the interface between the reconnection inflow and outflow, across which the plasma temperature increases drastically. It indicates that besides the Joule dissipation, the slow-mode shock may be another effective way to heat plasma.

\subsection{Parameter Dependence}

In our simulation, the most important parameters are the background magnetic field, the anomalous resistivity, and the height of the X-point (i.e., the reconnection site). In

order to show the influence of different parameters on the results, we performed extensive simulations by changing one parameter at a time with others being fixed.

Figure 8 shows the evolution of the magnetic reconnection rate for different initial background magnetic fields and anomalous resistivities, with the height of the initial $\mathrm{X}$-point being $1000 \mathrm{~km}$. It can be seen that the magnetic reconnection rate can reach the maximum much faster when we set a stronger initial magnetic field. It is also found that the large anomalous resistivity can shorten the evolution time from the initial stage to the time when the reconnection rate reaches the maximum. It should be noted here that, although the resistivity in the chromosphere is $\sim 3$ orders of magnitude larger than that in the corona, it is still too small from the simulation point of view. A so small resistivity would lead to a very thin diffusion layer near the reconnection X-point, which could not be resolved by the current numerical mesh. Therefore, the values of the anomalous resistivity we used are several orders of magnitude larger than the classical Spitzer resistivity (e.g., 
Kovitya \& Cram 1983).

Figure 9 shows the temperature distributions at the peak time of the magnetic reconnection rate. Our results indicate that the temperature enhancement is sensitive to the magnetic field. However, in all cases our results are consistent qualitatively with the

observed temperature enhancement in chromospheric microflares (Fang et al. 2006a). A quantitative comparison between the simulation and the observation will be described in Section 3.4.

\subsection{Scaling law}

The ranges of the variables such as the background magnetic field $\left(B_{0}\right)$, anomalous resistivity $\left(\eta_{0}\right)$ and the number density of hydrogen $\left(n_{H}\right)$ are very wide in observations. As a result, the maximum temperature enhancement $(\Delta T)$ and the reconnection lifetime $(\Delta t)$ may change greatly. In order to find a scaling law to relate $\Delta T$ and $\Delta t$ to the above-mentioned 3 variables, we change the variables one by one and perform a series simulations. As illustrated by Figure 10, the dataset can be well fitted by the following formula:

$$
\frac{\triangle T}{\triangle t} \approx 3.7\left(\frac{n_{H}}{3.4 \times 10^{19} \mathrm{~m}^{-3}}\right)^{-1.5}\left(\frac{B_{0}}{25 \mathrm{G}}\right)^{2.1}\left(\frac{\eta_{0}}{17.7 \Omega \mathrm{m}}\right)^{0.88} \mathrm{~K} \mathrm{~s}^{-1}
$$

From the energy point of view, as the reconnection proceeds, the Joule dissipation is consumed partly by the plasma radiation and ionization, with the rest being converted into the plasma heating. Therefore, we have

$$
n_{H} k \triangle T=\frac{J^{2}}{\sigma} \triangle t-n_{H} \alpha(y) n_{e} f(T) \triangle t+n_{H} \alpha(y)\left[n_{e} f(T)\right]_{t=0} \triangle t-\left[n_{e}-\left(n_{e}\right)_{t=0}\right] \chi_{H}
$$


where the expressions of $n_{e}, \alpha(y), f(T)$ are given by Equations (5) , (9) $)$, and (10). The items on the right-hand side of the Equation (15) are the Joule dissipation, the radiative

losses, the pre-heating and the ionization energy, respectively. If we assume that the plasma radiation and ionization are negligible, Equation (15) can be rewritten in a simple form as

$$
n_{H} k \triangle T \approx \frac{J^{2}}{\sigma} \triangle t=\eta \frac{\left(\nabla \times B_{0}\right)^{2}}{\mu_{0}^{2}} \triangle t \sim \eta \frac{B_{0}^{2}}{\mu_{0}^{2} L^{2}} \triangle t
$$

where $\mu_{0}$ is the vacuum permeability, $L$ is the scale of the reconnection region. Since in our simulation $L$ is fixed as a constant, we can get a simple theoretical formula as follow:

$$
\frac{\triangle T}{\triangle t} \propto \frac{B_{0}^{2} \eta}{n_{H}}
$$

It can be seen that the formula (14) given by our simulations is similar to the formula (17) except the relatively bigger deviation of the power index of $n_{H}$. The deviation is probably because the radiative loss and ionization items, which are related to $n_{H}$ but not to $B_{0}$ and $\eta_{0}$, have been considered in our simulations but are neglected in the analytical form. A further study will be done in order to find a more realistic scaling law in the future.

\subsection{Comparison with a Bright Microflare Event}

Fang et al. (2006a) analyzed five well-observed microflares and computed the thermal semi-empirical models for two typical microflares, one was bright and the other was faint. In oder to get the semi-empirical models, they first assume the temperature and turbulent velocity distributions, and then derive the density distribution. Based on these distributions they can calculate the $\mathrm{H} \alpha$ and Ca II $8542 \AA$ line profiles. The atmospheric model is adjusted until the calculated line profiles to match the observed ones. The temperature distribution of the bright microflares model is shown in the middle panel of Figure 11. In order to 
reproduce the temperature enhancement in the model, we set the initial background magnetic filed to be $300 \mathrm{G}$, the anomalous resistivity to be $159.3 \Omega \mathrm{m}$, and the initial reconnection height to be $500 \mathrm{~km}$. Since the magnetic field is strong, the total evolution time is short. The evolution of the reconnection rate, the temperature enhancement, and the velocity along the $y$-axis $\left(v_{y}\right)$ are shown in Figure 11 .

After $t=0.2 \tau_{A}$, the reconnection rate reaches about 0.1 . We choose the temperature distribution at $0.24 \tau_{A}$ to compare with the semi-empirical model. It is shown that there are two peaks in our simulation results. The smaller one is located at the height of 350 $\mathrm{km}$ and the bigger one is almost the same as the semi-empirical model and located at the height of $600 \mathrm{~km}$. The possible reason for this phenomenon is slow-mode MHD shock heating as shown in Figure 12. Below $800 \mathrm{~km}$, the temperature profile of our simulation is close to that of the semi-empirical model. However, above $800 \mathrm{~km}$, the temperature in the semi-empirical model becomes much higher than that of our simulation. The discrepancy might result from the fact that our simulations are performed for a relatively short period such that the reconnection jet has not yet reached the corona. Therefore, our result can only give a reasonable explanation for the temperature bump of the microflare mainly in the rise phase.

The right panel of Figure 11 shows the one-dimensional plot of $v_{y}$. From this figure it can be seen that the height of the reconnection X-point, which is characterized by the null velocity, has dropped from $500 \mathrm{~km}$ at $t=0$ to $450 \mathrm{~km}$ at $t=0.24 \tau_{A}$. The velocity of the upward flow is about $11.1 \mathrm{~km} \mathrm{~s}^{-1}$, which is much larger than that of the downward flow $\left(1.8 \mathrm{~km} \mathrm{~s}^{-1}\right)$. The downward jets can only reach the height of about $200 \mathrm{~km}$. 


\section{DISCUSSION AND SUMMARY}

More and more theoretical works have indicated that magnetic reconnection in the solar lower atmosphere can produce chromospheric microflares (Tandberg-Hanssen \& Emslie 1988; Liu et al. 2004; Fang et al. 2006a), as well as other small activities like Ellerman bombs (Fang et al. 2006b; Ding et al.|1998; Watanabe et al. 2008). With gravity, ionization and radiation being considered, we performed 2.5D MHD simulations. Since we have used the temperature distribution of the quiet-Sun atmospheric model VALC (Vernazza et al. 1981) and considered the helium abundance (10\%), our simulations are realistic to some extent.

In our simulation, we calculate the magnetic reconnection rate by using the definition $R=v_{\text {in }} / v_{A}$, rather than the formula $R=d \psi / d t$ used by Chen et al. (1999, 2001) and $R=\left|\eta J_{z}\right|$ used by Yokovama \& Shibata (2001), where $\psi$ means the magnetic flux function and $J_{z}$ stands for the $z$-component of current density. If we use the formula $R=d \psi / d t$ or $R=\left|\eta J_{z}\right|$, the reconnection rate has a large pulse, which can be greater than 1.0 at the beginning of the evolution. It is non-physical, as mentioned by Yokoyama \& Shibata (2001). However, it should be noted that all of these methods can give a similar result after enough time of evolution.

In the subsections 3.1 and 3.4, we studied the magnetic reconnection with the X-point at different heights, e.g., $500 \mathrm{~km}$ and $1000 \mathrm{~km}$. Our results, in the case of $500 \mathrm{~km}$, can well reproduce the temperature enhancement in the semi-empirical model (Fang et al. 2006a). At the heights of $500 \mathrm{~km}$ and $1000 \mathrm{~km}$, the temperature enhancement is 1000-2000 K and 2000-3000 K, respectively. In the case of $1000 \mathrm{~km}$, we found that the height of X-point can move up by tens of kilometers. This is different from the results of the case of $500 \mathrm{~km}$, where the X-point moves down. It is noted that the temperature enhancement we got in our simulations is several thousand Kelvin, and our results can only represent the rise phase 
of chromospheric microflares since our simulations were performed only until the upward reconnection jet reaches the bottom of the transition region. Of course, those microflares with strong EUV, X-ray, and microwave emissions may occur at the lower corona. In this case, the emissions in X-rays, EUV and microwave can be naturally explained.

It is worth noting that our simulations have some limitations. Even though we limited the computational height below $2000 \mathrm{~km}$, the plasma $\beta$ at the bottom boundary is still six orders of magnitude larger than that at the top boundary. It causes some numerical instability in our simulations. Besides, our initial magnetic configuration is relatively simple. Moreover, as it is well known, the current sheet should be very thin in the real situation, e.g., less than hundreds of meters, while in our simulation the minimum grid sizes are $\triangle x=1.25 \mathrm{~km}$ and $\triangle y=5 \mathrm{~km}$, which are too large to simulate the current sheet. All these contribute to the fact that our simulations can not reproduce the real observations in details. Further improvement of this work is expected.

In summary, we give the conclusions as follows:

1. Our 2.5D MHD simulations can reproduce the temperature enhancement in chromospheric microflares qualitatively. The temperature increase in the cases when the reconnection point is at the height of $500 \mathrm{~km}$ and $1000 \mathrm{~km}$ can reach 1000-2000 K and 2000-3000 K, respectively.

2. The free parameters in our $2.5 \mathrm{D}$ simulation are the background magnetic field $\left(B_{0}\right)$ and the anomalous resistivity $\left(\eta_{0}\right)$. We have found that the temperature enhancement is sensitive to the background magnetic field, while the total evolution time is sensitive to the magnitude of anomalous resistivity.

3. Our simulation results indicate that the velocity of the upward jet is much larger than that of the downward jet, and the X-point may exhibit downward or upward motions. 
4. We have performed a parameter survey, and found that the temperature enhancement and the total evolution time in the chromospheric reconnection follow the scaling law:

$$
\frac{\triangle T}{\triangle t} \approx 3.7\left(\frac{n_{H}}{3.4 \times 10^{19} \mathrm{~m}^{-3}}\right)^{-1.5}\left(\frac{B_{0}}{25 \mathrm{G}}\right)^{2.1}\left(\frac{\eta_{0}}{17.7 \Omega \mathrm{m}}\right)^{0.88} \mathrm{~K} \mathrm{~s}^{-1}
$$

The computations were done by using the HP ProLiant BL260C G5 Blade system at the Department of Astronomy of Nanjing University of China. This work is supported by the National Natural Science Foundation of China (NSFC) under the grant numbers 10221001, 10878002, 10403003, 10620150099, 10610099, 10933003 and 10673004, as well as the grant from the 973 project 2006CB806302. 


\section{REFERENCES}

Brosius, J. W., \& Holman, G. D. 2009, ApJ, 692, 492

Brown, J. C. 1973, Sol. Phys., 31, 143

Chen, P. F., Fang, C., Tang, Y. H., \& Ding, M. D. 1999, ApJ, 513, 516

Chen, P.-F., Fang, C., \& Ding, M.-D. D. 2001, ChJAA, 1, 176

Cramer, N. F. \& Donnelly, I. J. 1979, PASAu, 3, 367

Ding, M. D., Henoux, J.-C., \& Fang, C. 1998, A\&A, 332, 761

Emslie, A. G., \& Noyes, R. W. 1978, Sol. Phys., 57, 373

Fang, C., Tang, Y.-H., \& Xu, Z. 2006a, ChJAA, 6, 597

Fang, C., Tang, Y. H., Ding, M. D., \& Chen, P. F. 2006b, ApJ, 643, 1325

Gan, W. Q., \& Fang, C. 1990, ApJ, 358, 328

Gary, D. E., \& Zirin, H. 1988, ApJ, 329, 991

Golub, L., Krieger, A. S., Silk, J. K., Timothy, A. F., \& Vaiana, G. S. 1974, ApJ, 189, L93

Golub, L., Krieger, A. S., Harvey, J. W., \& Vaiana, G. S. 1977, Sol. Phys., 53, 111

Kovitya, P., \& Cram, L. 1983, Sol. Phys., 84, 45

Kudoh, T., Matsumoto, R., \& Shibata, K. 1999, Comput. Fluid Dynamics J., 8, 56.

Lin, R. P., Schwartz, R. A., Kane, S. R., Pelling, R. M., \& Hurley, K. C. 1984, ApJ, 283, 421

Liu, C., Qiu, J., Gary, D. E., Krucker, S., \& Wang, H. 2004, ApJ, 604, 442 
Machado M. E., Avrett E. H., Vernazza J. E. et al. 1980, ApJ, 242, 336

Ning, Z. 2008, ApJ, 686, 674

Nishizuka, N., Shimizu, M., Nakamura, T., Otsuji, K., Okamoto, T. J., Katsukawa, Y., \& Shibata, K. 2008, ApJ, 683, L83

Petschek, H. E., 1964, in physics of Solar Flares, ed. W. N. Hess, NASA SP-50, Washington, DC, p. 425

Porter, J. G., Toomre, J., \& Gebbie, K. B. 1984, ApJ, 283, 879

Qiu, J., Liu, C., Gary,D. E., Nita, G. M., \& Wang, H. 2004, ApJ, 612, 530

Shimizu, T., Shine, R. A., Title, A. M., Tarbell, T. D., \& Frank, Z. 2002, ApJ, 574, 1074

Takeuchi, A., \& Shibata, K. 2001, ApJ, 546, L73

Tandberg-Hanssen, E., \& Emslie, A. G. 1988, The physics of solar flares, (Cambridge: Cambridge Uni. Press)

Tang, Y. H., Li, Y. N., Fang, C., Aulanier, G., Schmieder, B., Demoulin, P., \& Sakurai, T. 2000, ApJ, 534, 482

Vernazza, J. E., Avrett, E. H., \& Loeser, R. 1981, ApJS, 45, 635

von Rekowski, B., \& Hood, A. W. 2008, MNRAS, 385, 1792

Watanabe, H., et al. 2008, ApJ, 684, 736

Xia, C., Fang, C., Chen, Q. R., \& Tang, Y. H. 2007, Adv. Space Res., 39, 1402

Yokoyama, T., \& Shibata, K. 2001, ApJ, 549, 1160 
Table 1: Normalization Units

\begin{tabular}{cccc}
\hline \hline Variable & Quantity & Unit & Value \\
\hline$x, y$ & Length & $L_{0}$ & $2000 \mathrm{~km}$ \\
$T$ & Temperature & $T_{0}$ & $6000 \mathrm{~K}$ \\
$\rho$ & Density & $\rho_{0}$ & $6.0 \times 10^{-8} \mathrm{~kg} \mathrm{~m}^{-3}$ \\
$p$ & Pressure & $p_{0}=\rho_{0} T_{0} \kappa_{B} / m_{H}$ & $2.97 \mathrm{~N} \mathrm{~m}^{-2}$ \\
$\mathbf{V}$ & Velocity & $V_{0}=\left(T_{0} \kappa_{B} / m_{H}\right)^{1 / 2}$ & $7.035 \mathrm{~km} \mathrm{~s}^{-1}$ \\
B & Magnetic field & $B_{0}=\left(\mu_{0} \rho_{0} T_{0} \kappa_{B} / m_{H}\right)^{1 / 2}$ & $19.3 \mathrm{G}$ \\
$t$ & Time & $t_{0}=L_{0}\left(T_{0} \kappa_{B} / m_{H}\right)^{-1 / 2}$ & $284 \mathrm{~s}$ \\
\hline
\end{tabular}




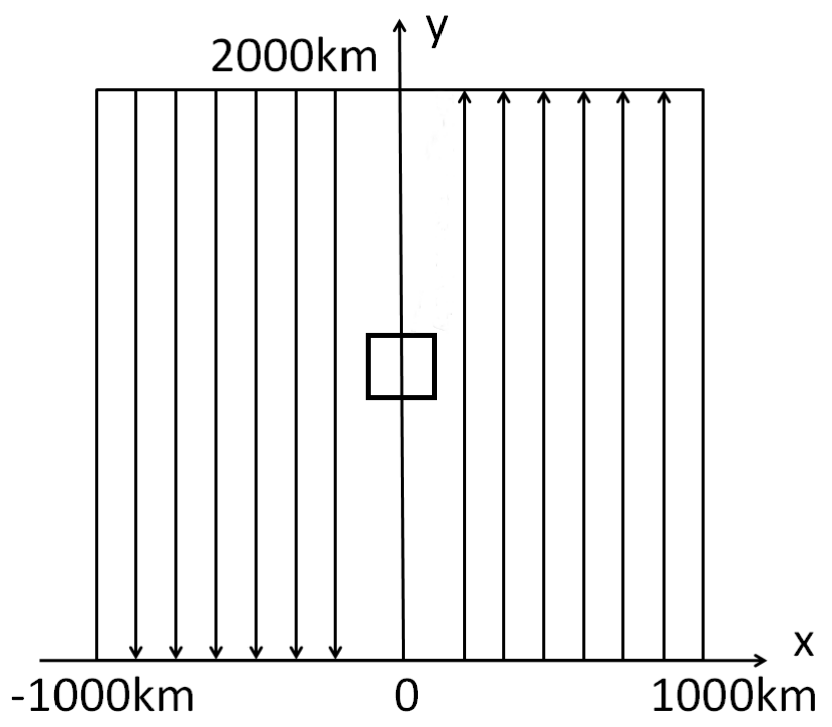

Fig. 1.- Initial magnetic field configuration. The range of the computational box is from $-1000 \mathrm{~km}$ to $1000 \mathrm{~km}$ in the $x$ direction and from 0 to $2000 \mathrm{~km}$ in the $y$ direction. 

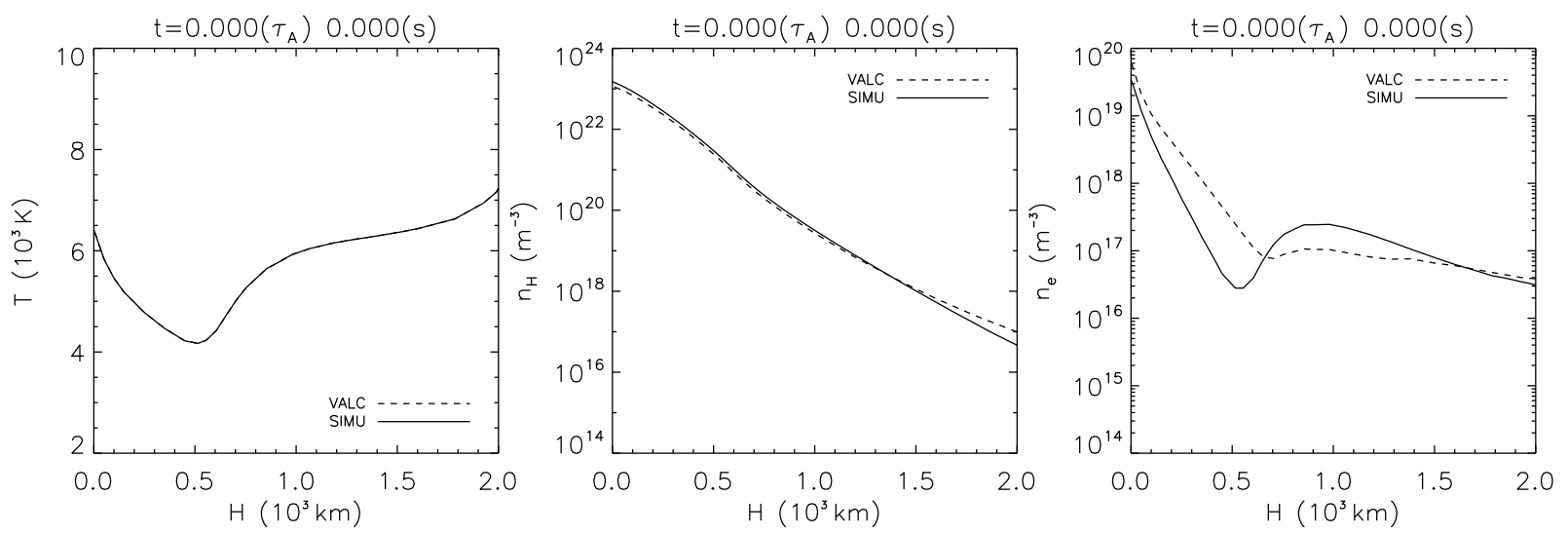

Fig. 2.- Initial distributions of the temperature (left panel), the number density of hydrogen (middle panel), and the electron number density (right panel). The distributions in the quietSun atmospheric model VALC (dashed lines) are also shown. 

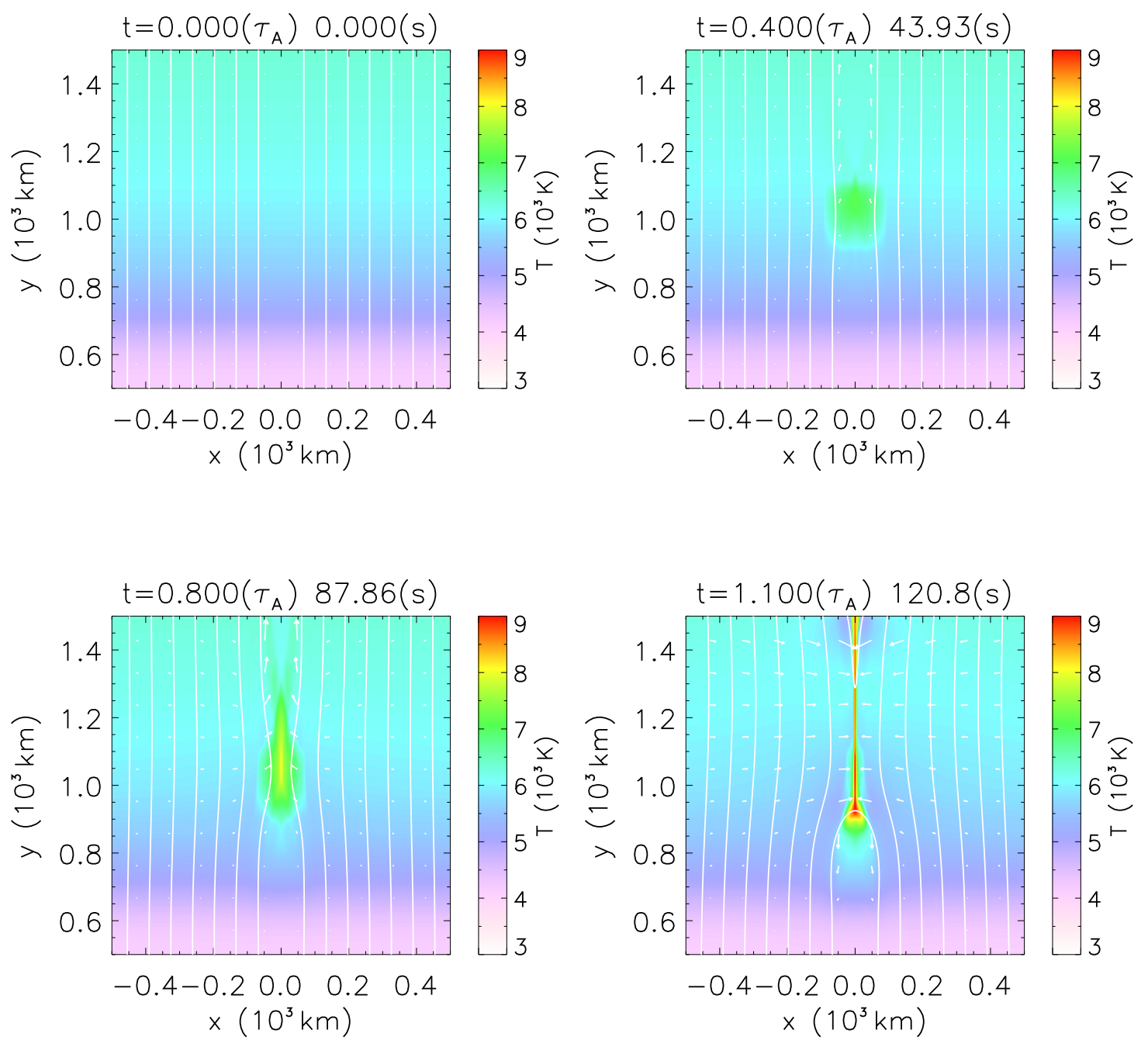

Fig. 3.- Temperature distributions (color scale), projected magnetic field (solid lines) and velocity field (vector arrows) at $t=0$ and $t=0.4$ (upper panels) and $t=0.8$ and $t=1.1 \tau_{A}$ (lower panels). 


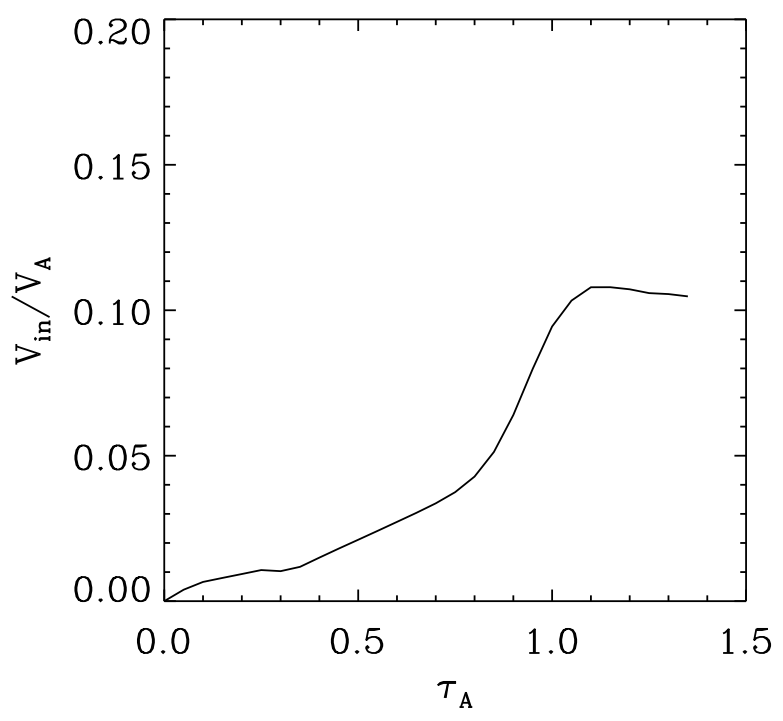

Fig. 4.- Evolution of the magnetic reconnection rate $\left|V_{i n}\right| / V_{A}$. The rate reaches the maximum at the time of $1.1 \tau_{A}(120.8 \mathrm{~s})$. 


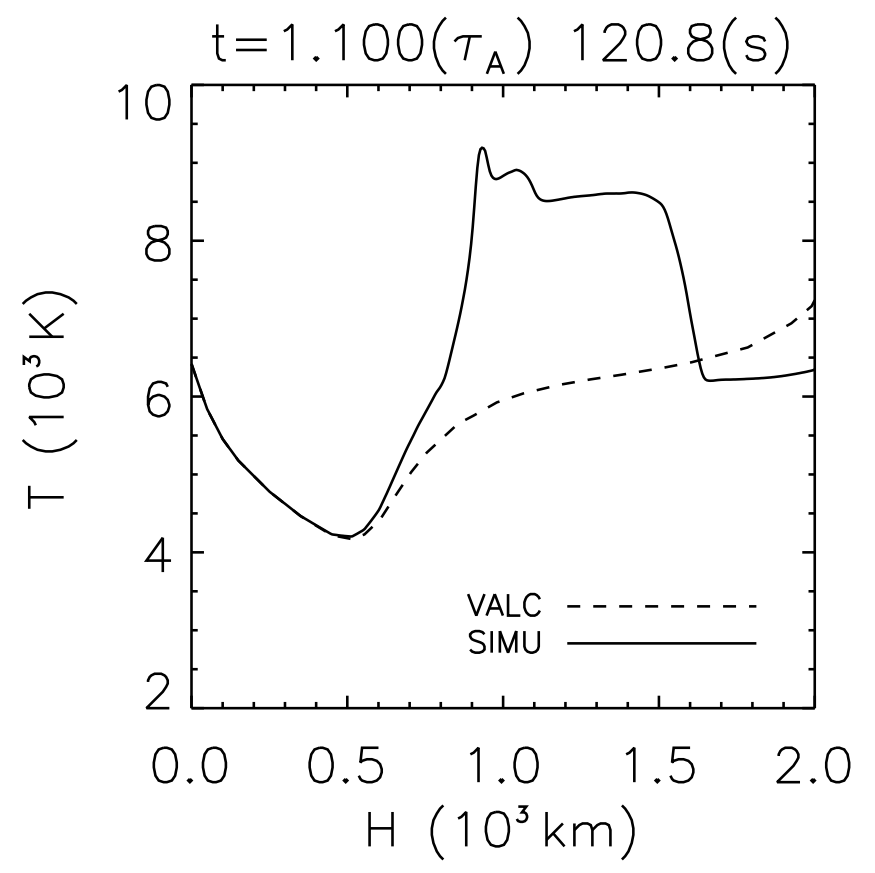

Fig. 5.- One-dimensional $T$ plot (solid line) along the y-axis at the time of the maximum reconnection rate $\left(1.1 \tau_{A}\right)$. The initial temperature plot (dashed line), i.e. VALC temperature distribution, is also shown. 


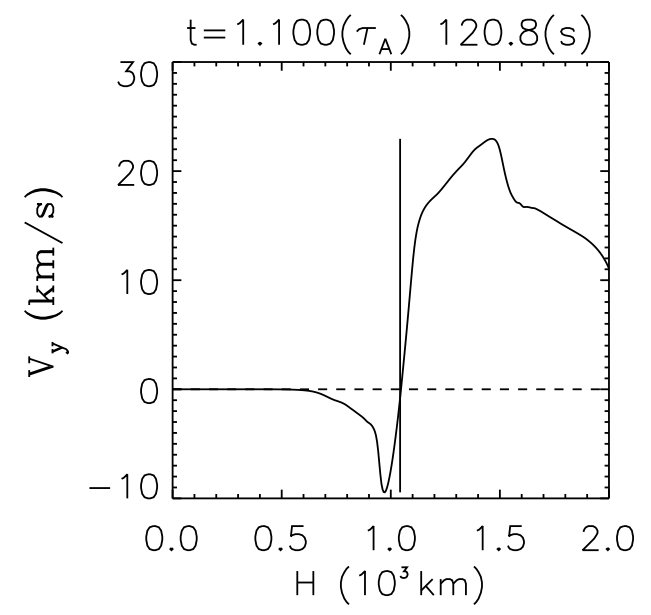

Fig. 6.- One-dimensional $V_{y}$ plot (solid line) along the y-axis at the time of maximum reconnection rate $\left(1.1 \tau_{A}\right)$. The height of the $\mathrm{X}$-point is marked by a vertical line. The initial $V_{y}$ plot (dashed line) is also shown. 

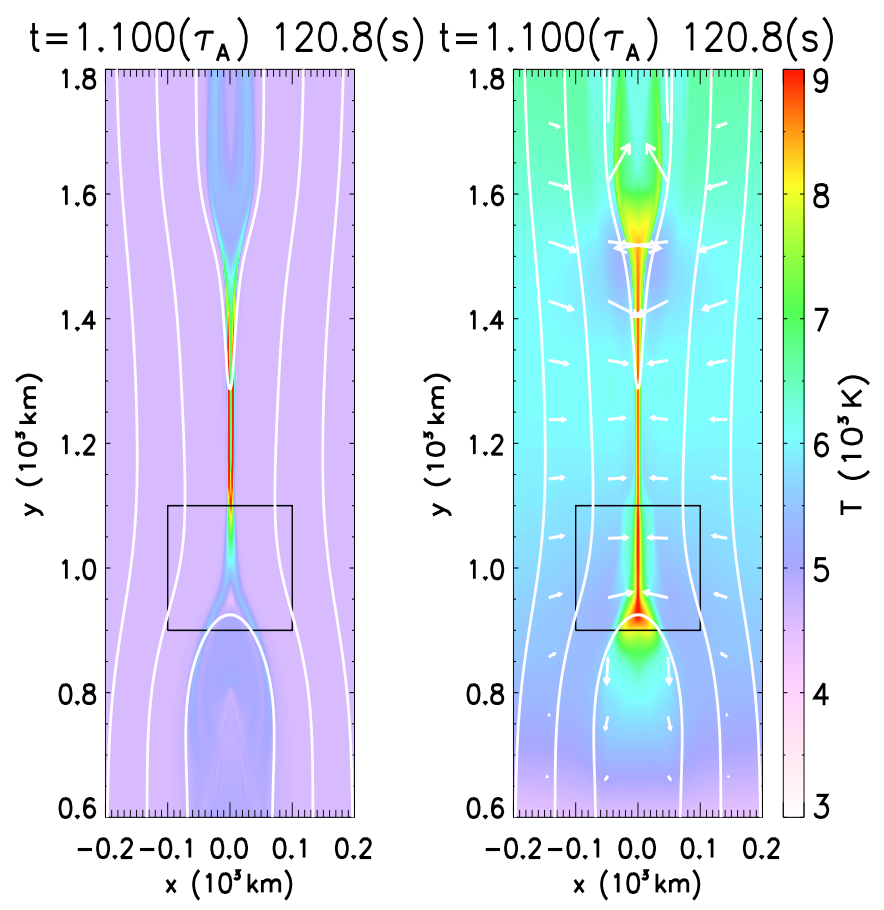

Fig. 7.- Z-component of the current density (left panel) and temperature distributions (right panel) at the time of the maximum reconnection rate $\left(1.1 \tau_{A}\right)$. There is no need to plot the color bar for the left panel which based on dimensionless data, but in both panels the same color table is used, which means that the red side stands for higher current density and temperature, while the white side stands for the lower ones. 

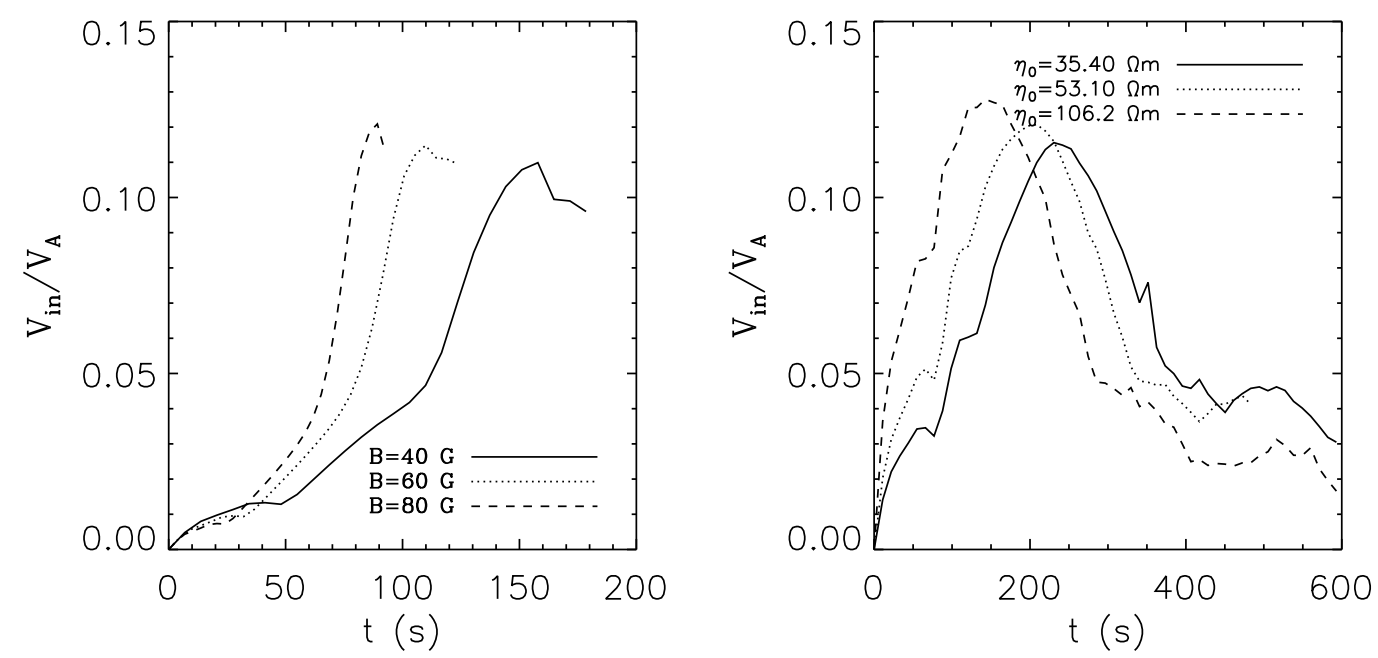

Fig. 8. - Evolution of the magnetic reconnection rate for different initial background magnetic fields $(B)$ and anomalous resistivity $\left(\eta_{0}\right)$. The height of the initial X-point is 1000 $\mathrm{km}$. 

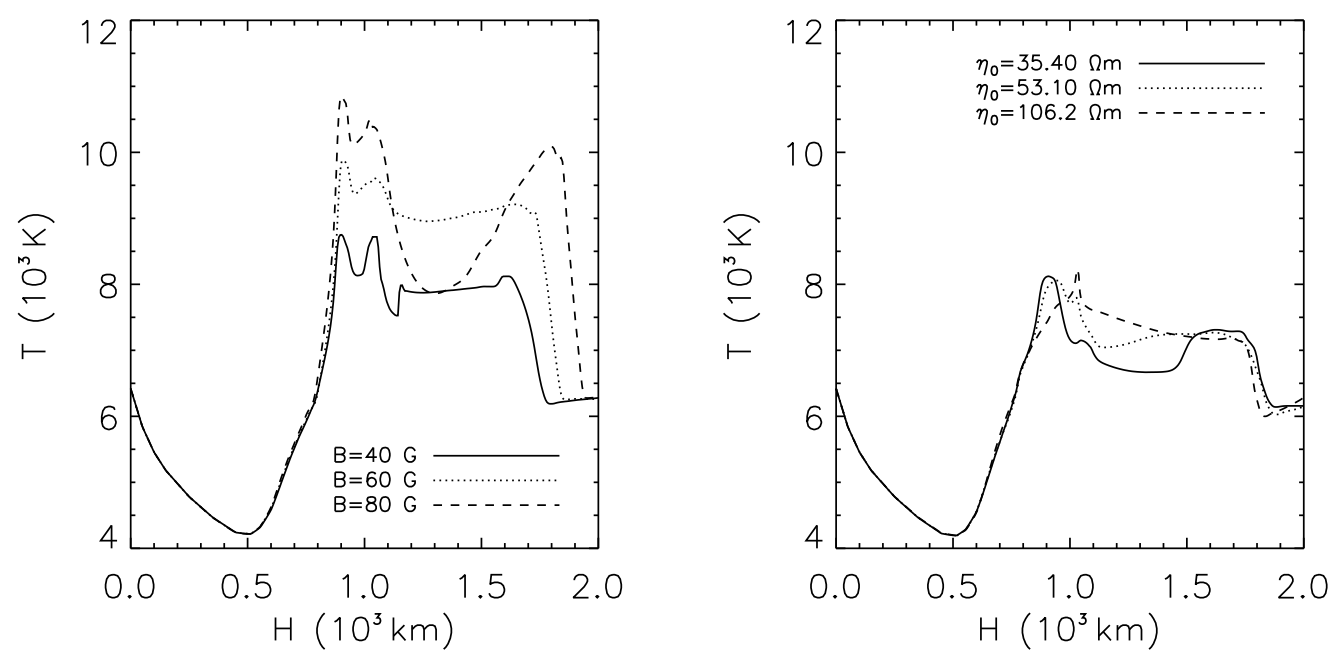

Fig. 9.- Temperature distributions for different initial background magnetic field $(B)$ and anomalous resistivity $\left(\eta_{0}\right)$ at the time of maximum rate of magnetic reconnection which have been shown in Figure 8, The height of the initial X-point is $1000 \mathrm{~km}$. 

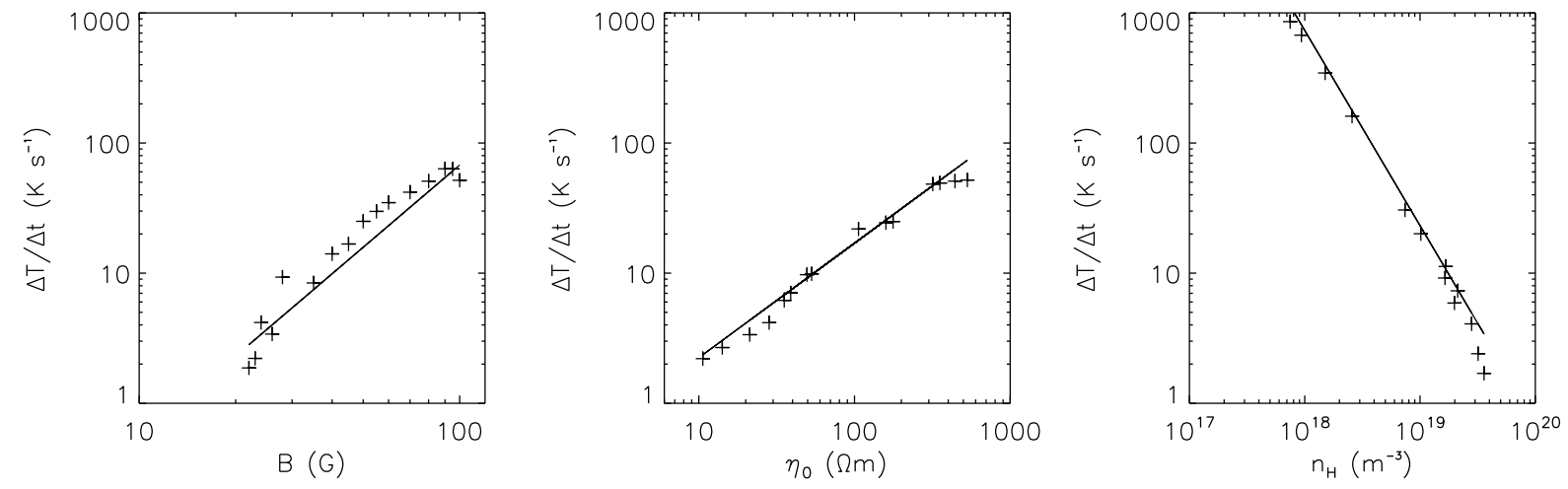

Fig. 10.- Temperature enhancement per unit time as a function of the background magnetic field $B$ (left panel), the anomalous resistivity $\eta_{0}$ (middle panel), and the number density of hydrogen atom $n_{H}$ (right panel). In the left panel, the initial reconnection height is 1000 $\mathrm{km}$ and the anomalous resistivity is $17.7 \Omega \mathrm{m}$. In the middle panel, the initial reconnection height is $1000 \mathrm{~km}$ and the magnetic field is $25 \mathrm{G}$. In the right panel, the anomalous resistivity is $17.7 \Omega \mathrm{m}$ and the magnetic field is $25 \mathrm{G}$. 

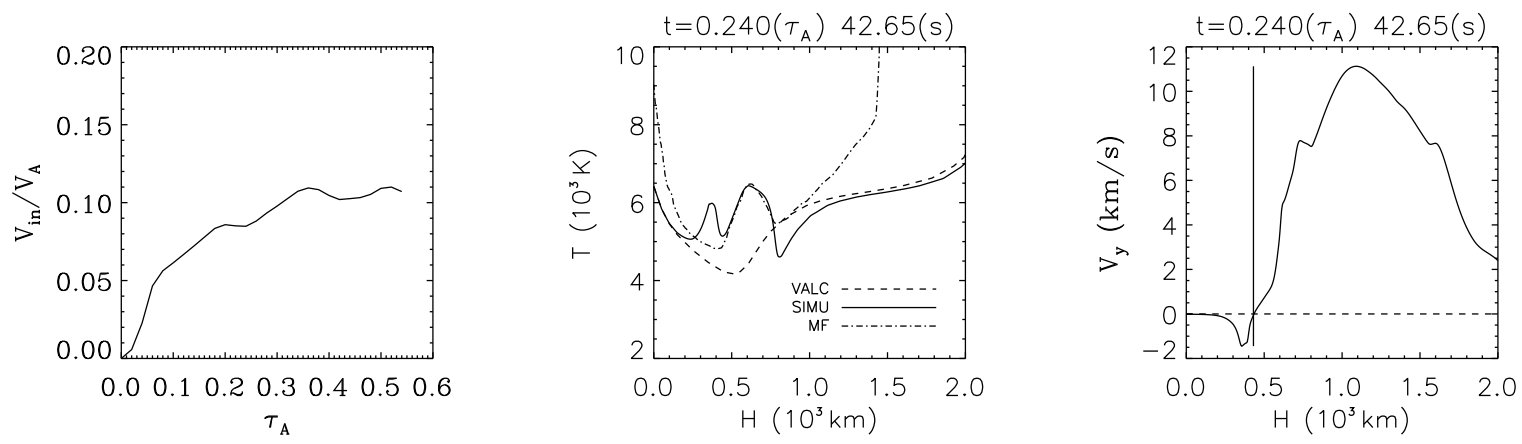

Fig. 11. - Magnetic reconnection rate (left panel), $T$ (middle panel) and $V_{y}$ (right panel) plot (solid line) at the time $0.24 \tau_{A}$. The initial conditions of $T$ and $V_{y}$ are also shown (dashed lines). The height of X-point is marked by the vertical line in the right panel and the temperature distribution of bright microflare $(\mathrm{MF})$ is given by dash-dotted line in the middle panel. 

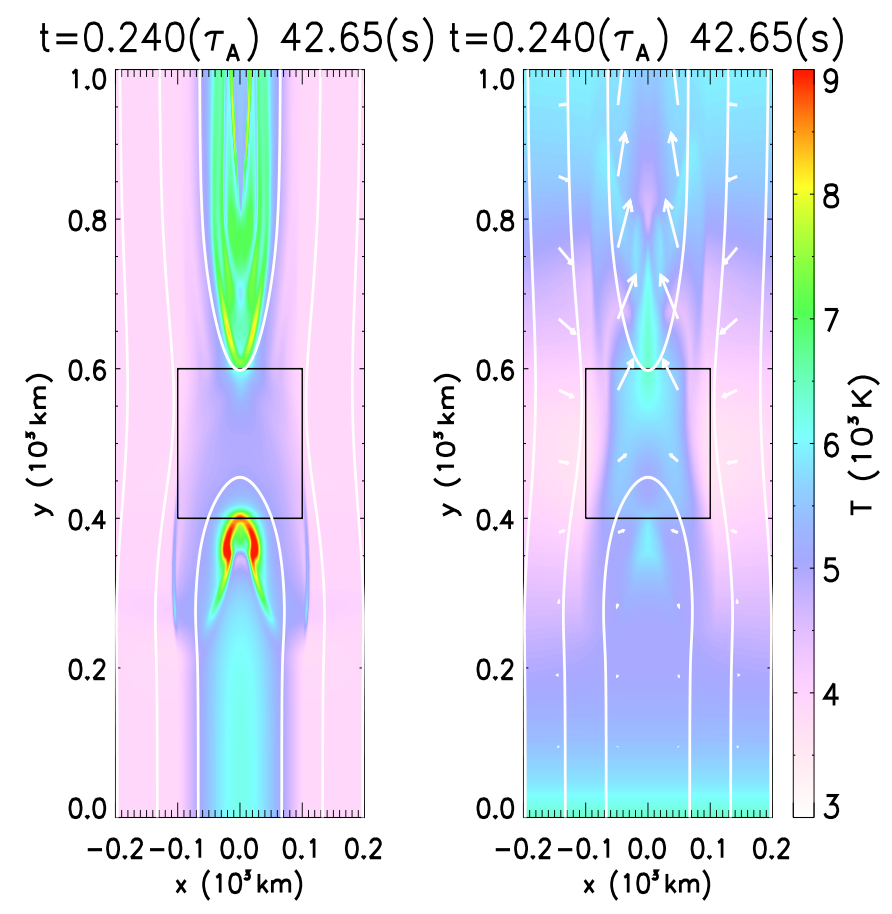

Fig. 12.- Z-component of current density (left panel) and temperature distributions (right panel) at the time of maximum reconnection rate $\left(0.24 \tau_{A}\right)$. Both panels have used the same color table, which means that the red side stands for higher current density and temperature, the white side stands for the lower ones. 